Check for updates

London, UK

Cite this as: BMJ 2020;369:m2441 http://dx.doi.org/10.1136/bmj.m2441 Published: 17 June 2020

\title{
WHO warns that averting flu pandemic may be harder as surveillance switches to covid-19
}

\section{Jonathan Owen}

The demands of responding to covid-19 are damaging the prospects of developing effective influenza vaccines and reducing the chances of preventing a flu pandemic, according to the World Health Organization.

More than 500 million people are vaccinated against influenza every year. The composition of flu vaccines is based on recommendations from WHO, drawing on data from its Global Influenza Surveillance and Response System to select specific strains to target.

Tedros Adhanom Ghebreyesus, WHO director general, said, "Despite the ongoing global response to the covid-19 pandemic, we cannot lose sight of other significant public health problems, including influenza."

Speaking during a press conference at WHO's headquarters in Geneva on 15 June, he warned, "Influenza surveillance has either been suspended or is declining in many countries, and there has been a sharp decline in sharing of influenza information and viruses because of the covid-19 pandemic."

Global and national systems for monitoring and tackling influenza have been focused on responding to the threat of covid-19 and there has been a "dramatic decrease in the number of specimens tested for influenza globally" compared to the past three years, said Tedros.

The number of virus shipments to WHO collaborating centres has fallen by $62 \%$ and there has been a $94 \%$ drop in the number of influenza viruses with genetic sequence data being submitted to the Global Initiative on Sharing All Influenza Data, he added.

"These decreases are because of a combination of matters, including the repurposing of staff and supplies, overburdened laboratories, and transport restrictions," he said.

"These disruptions may have short and long term effects, such as the loss of capacities to detect and report new influenza viruses with pandemic potential."

WHO is calling on countries to integrate testing for covid-19 with routine influenza surveillance.

"This is not only cost effective, it's also essential for protecting the world against the next flu season," Tedros said. "As we enter the southern hemisphere influenza season and begin planning for the northern hemisphere season, we must ensure that influenza remains a top priority."

Stressing the need for urgency, he added, "There is no time to lose."

Responding to the concerns, Jamie Lopez Bernal, head of flu at Public Health England, said, "The covid-19 pandemic presents a range of challenges to surveillance of influenza and other respiratory diseases and this is being closely monitored. We are continuing to produce regular flu surveillance reports and surveillance for the two diseases is closely integrated."

He added, "It is essential that laboratories continue to refer samples to the national reference lab for influenza testing and characterisation to support national and global surveillance."

Bharat Pankhania, an expert in infectious disease management at the University of Exeter's College of Medicine and Health, told The BMJ, "While we have a lot of knowledge about influenza it doesn't mean it has been conquered and influenza viruses have just as much potential for emerging as pandemic strains.” 